\title{
Source cuing: Memory for melodies
}

\author{
GABRIEL A. RADVANSKY and JULE K. POTTER \\ University of Notre Dame, Notre Dame, Indiana
}

\begin{abstract}
Source cuing is a source-monitoring process in which the retrieval of a memory trace is aided by the use of a memory probe that includes information that is indicative of the original source. This is in contrast to source discrimination, where people need to retrieve the identity of the source of information. Thus, in source cuing, the source information is given, and in source discrimination, the source information is to be retrieved. The operation of source cuing was demonstrated in two experiments in which people had to identify which of two melodies had been heard earlier. Source cuing was present for information that was more indicative of the source (i.e., timbre), but not for information that was less indicative of the source (i.e., pitch). A third experiment demonstrated that the use of source cuing can be influenced by the retrieval context.
\end{abstract}

Over the past several years, there has been a great deal of research on the use of source information in memory (for a review, see Johnson, Hashtroudi, \& Lindsay, 1993). The aim of the bulk of this research has been to test people's ability to monitor the source of information that they retrieve from memory, which is done either in the service of making recognition decisions or to retrieve source information itself. For example, if an episode is remembered, the person is tested for knowledge of where the information came from (e.g., whether person X or Y said a word, whether an action was observed or imagined, etc.). This process of identifying the source of information is referred to here as source discrimination. The present research takes a different approach. The issue addressed here is whether information about source can influence the ability to retrieve a memory of an event. That is, can memory performance be affected by whether features in a memory probe are consistent or inconsistent with the original source in which the information was encoded? The use of source information in memory retrieval is called source cuing. Thus, whereas in source discrimination source information is to be retrieved, in source cuing it is part of the memory probe used to access the memory trace in the absence of any instruction to use or report on the source information. More generally, the issue addressed here is whether source information is used differently in memory cuing than is other stimulus attribute information.

To test this aspect of memory, we need a task in which source information can be easily identified. Probes that have source cues that match the original event would pre-

Part of this research was presented at the 70th meeting of the Midwestern Psychological Association in May 1998, Chicago. This research was supported in part by an NIMH B/START award to the first author. Correspondence concerning this article should be addressed to G. A. Radvansky, Department of Psychology, University of Notre Dame, Notre Dame, IN 46556 (e-mail: radvansky.1@nd.edu). sumably result in better memory performance than would probes with different source cues. To this end, we elected to test a person's memory for melodies with a method used by Wolpert (1990) and improved upon by Radvansky, Fleming, and Simmons (1995). In these studies, a person first heard a short melody. This melody was presented in a specific timbre, such as a piano. After a brief $(30-\mathrm{sec})$ distractor period, the listener was presented with a twoalternative forced-choice recognition test. One melody was the original one, and the other was a new melody. In the Wolpert and the Radvansky et al. studies, one of the alternatives was in the same timbre as the original melody (e.g., a piano), and the other was in a different timbre (e.g., a vibraphone). The critical manipulation was whether the target melody was in the same timbre as the original (match) or was in a different timbre (mismatch). For example, on a mismatch trial, if the original melody had been played on a piano, for the recognition test, the target melody would be presented on a vibraphone, and the new distractor melody would be presented on a piano. The findings indicated that people were more likely to make a recognition error in the mismatch condition, relative to the match condition. That is, listeners' memory performance showed a dependence on the relationship between the timbre in which the melody was originally presented and the timbre of that melody on a subsequent memory test (for a related finding, see Peretz, Gaudreau, \& Bonnel, 1998, Experiment 3).

Radvansky et al. (1995) suggested two potential explanations for this finding. One was that timbre was used as source information to help retrieve the memory trace. The basic idea is that timbre carries information that readily identifies the source. The unique acoustical features of each timbre indicate a distinct source from which the melody originated. Different instruments are different entities, often played by different people, and hence are different sources. Melodies in the same timbre are more likely to have come from the same source (both played on a piano) than melodies in a different timbre (one on a piano 
Table 1

Design of the Experiments for Both Timbre and Pitch

\begin{tabular}{|c|c|c|c|c|}
\hline \multicolumn{2}{|c|}{ Dimension Match-Same } & \multirow[t]{2}{*}{ Match-Different } & \multirow[t]{2}{*}{ Mismatch-Same } & \multirow[t]{2}{*}{ Mismatch-Different } \\
\hline Timbre & & & & \\
\hline Original & piano & piano & piano & piano \\
\hline Target & piano & piano & vibes & vibes \\
\hline Distractor & piano & vibes & vibes & piano \\
\hline \multicolumn{5}{|l|}{ Pitch } \\
\hline Original & Key 1 & Key 1 & Key 1 & Key 1 \\
\hline Target & Key 1 & Key 1 & Key 2 & Key 2 \\
\hline Distractor & Key 1 & Key 2 & Key 2 & Key 1 \\
\hline
\end{tabular}

and one on a vibraphone). Different timbres are different musical voices. This is akin to having different people read different sentences. This voice information is known to be linked with the memory trace and can affect recognition performance (Mullennix, Pisoni, \& Martin, 1989; Palmeri, Goldinger, \& Pisoni, 1993). Thus, when the source of the information in the memory probe matches the original, it is easier to access the memory trace containing the melody than when it is different. The other potential explanation was that there was a forgetting of stimulus attributes (e.g., Riccio, Rabinowitz, \& Axelrod, 1994), in which the poorer memory performance was due to generalized differences in any feature of the stimulus, not necessarily those related to source.

In the melody recognition paradigm, we assumed that timbre, like the acoustical features attributed to vocal tract differences in speech, is a relatively clear indicator of source information. This is because different timbres are associated with different entities in the world. Many musical instruments produce acoustic features that are very distinctive, relative to others. Cues that more clearly provide information about a source, such as timbre, are referred to as high-source cues. However, other features do not communicate information about source in such a clear fashion. An example of this would be the pitch of a melody. Information about pitches in a melody is akin to information about vocal tone quality in speech, such as whether a speaker is whispering or shouting. If a melody were transposed to a different key, within a reasonably close range, ${ }^{1}$ this alone would serve as a poor means of discriminating source. This is because shifts in pitch, if timbre is kept constant, can easily come from the same entity. Cues that do not provide clear information about a source are referred to as low-source cues. In general, we expect a different pattern of results for high- and lowsource information in terms of their influence on a later recognition test.

In addition to the idea that timbre and pitch will have different cuing effects, these two features of melodies were selected because of their salience in music. Specifically, pitch and timbre are both used to chunk information in heard melodies. For example, when there is shift from one to another during the presentation of a melody, people will identify what follows as being part of a new unit. Similarly, people find it easier to identify interleaved melodies that are segregated by timbre or pitch than those segregated by other features, such as changes in pitch envelope or adding effects like reverb (see, e.g., Hartmann \& Johnson, 1991).

One way to view this issue is to suggest that this is a form of encoding specificity (e.g., Tulving \& Thompson, 1973). This is the idea that memory performance is better when the context is the same at retrieval as at encoding. However, we think that source cuing goes further in that it suggests that some contexts (features) are more effective in aiding memory retrieval than are others. Moreover, the effectiveness of various features can be identified on the basis of the role they play in the retrieval process. If the melody itself is considered the target item to be retrieved, timbre and pitch can be considered the context in much the same way the color, location, or font of a word would be in a verbal learning task. However, these different types of "context" are predicted to have different effects on memory performance. When the context more clearly provides information about sourceinformation about where the melody came from-it may be able to serve as a more effective retrieval cue.

In Experiments 1 and 2, we used a modification of the design used by Radvansky et al. (1995). In the Radvansky et al. study, the alternatives on the recognition test always differed on one critical dimension (timbre). That is, one recognition choice was in the same timbre as the original, and the other choice was in a different timbre. In the present Experiments 1 and 2, half of the recognition test pairs were constructed in this way. However, for the other half, both the target and the distractor were the same on that dimension (e.g., of the same timbre). For half of those latter pairs, both were the same as the original melody on the critical dimension, and for the other half, both differed. An illustration of all four conditions is presented in Table 1. Radvansky et al. used conditions that corresponded only to the third and fifth columns. The addition of trials in which the target and the distractor are the same on the critical dimension corresponds to the addition of the second and fourth columns.

Thus, this is a $2 \times 2$ design in which target type (match or mismatch with the original melody) was crossed with distractor type (same as or different from the target). Conditions in this design will be referred to by the combination of target type and distractor type. For example, 
match-same means that, on the critical dimension, the target matches the original and the distractor has the same feature as the target.

\section{PREDICTIONS}

The notion of source cuing provides some predictions for the pattern of results in this design. What is of interest here are those cases in which a person is not able to access the original memory trace of the melody via other information in the probe (e.g., the melodic contour) but can use the critical feature as a retrieval cue. (Obviously, for those cases in which a memory trace of the melody can initially be accessed, that would be used.) The degree to which different features are able to serve as source cues should result in different patterns of retrieval performance.

First, consider the case in which timbre is the critical dimension. If source information (timbre) is used to access the memory trace of the other content (melody), performance will be better when the target timbre matches the original than when it mismatches. For the match trials (both match-same and match-different), the timbre of the target probe can be used to access a memory trace of the timbre of the original melody. This information is associated with the memory trace for the melody, which is then retrieved and compared with the probe melody, resulting in a positive response. However, for the mismatch trials, this cannot occur. For the mismatch-same condition, the timbre of both memory probes is different from the original and so cannot access the trace for the timbre information. Thus, the person must guess between the alternatives. For the mismatch-different condition, the timbre of the distractor probe will access the timbre information in memory. However, because the associated melody is not the same as the one in the probe, a positive response cannot be made on the basis of this information. Again, not being able to retrieve the needed melody information, the person is left to pick between two alternatives at chance. Thus, the predicted pattern of error rates is match-same $=$ match-different $<$ mismatch-same $=$ mismatch-different. The type of distractor (same vs. different) should have no effect. This pattern cannot be assessed from the Radvansky et al. (1995) and Wolpert (1990) data, because distractor type was not manipulated.

Now consider the prediction for when pitch is the critical dimension. In the context of our experiments, as was described earlier, pitch is considered to be less effective for discriminating source. As such, memory for melody and pitch would not be highly associated in memory but would be stored more independently. Thus, pitch would be a poorer cue for retrieving a trace of the melody. For the match-different condition, the pitch of the target would correspond to the pitch in memory, but the pitch of the distractor would not. The use of this information would lead to a correct response, even if the melody information was never retrieved. For the mismatch-different condition, the pitch of the distractor would correspond to the pitch memory. Using this alone would lead to an incorrect response. For the match-same condition, both probes would access the pitch memory. However, because this information does not discriminate between the two, correct responses would be made about half the time. Finally, for the mismatch-same condition, neither probe would access the pitch memory. Again, there is no means of discriminating between the two choices, and so correct responses are made about half the time. This selection would be correct half of the time. Thus, the predicted pattern of error rates is match-different $<$ match-same $=$ mismatch-same $<$ mismatch-different.

Timbre was manipulated in Experiment 1, and pitch was manipulated in Experiment 2 . If different patterns of error rates were to be observed in Experiments 1 and 2, this would be consistent with there being a difference between how high- and low-source information cues are used during retrieval. However, if the same pattern of results were to be observed, this would be consistent with a view according to which any change in an event feature results in poorer memory and there is nothing particularly special about source information.

In all of the experiments reported here, only nonmusicians were tested. This is because Wolpert (1990) and Radvansky et al. (1995) found that the performance of musicians is much closer to ceiling, making is harder to detect differences, although the musicians show a pattern of errors similar to that for nonmusicians. For our purposes, we defined people as being nonmusicians if they had 3 or fewer years of formal music training.

\section{EXPERIMENT 1 Timbre}

\section{Method}

Listeners. Sixty-four people, with from 0 to 3 years $(M=1.2$, $S D=1.0$ ) of musical experience, were tested in Experiment 1 and given partial class credit in exchange for their participation.

Apparatus. This study was conducted on IBM-compatible computers equipped with Soundblaster 16-bit ASP and Waveblaster sound cards. This ensured sufficient control over melody production, as well as reasonably high quality sounds. The melodies were amplified by a Fostex PH-5 headphone amplifier and presented over Sony MDR-7506 headphones.

Melodies. The melodies used in all three experiments were the same as those used by Radvansky et al. (1995). These tunes were chosen from Easy Classics to Moderns (Agnay, 1956), a book of relatively simple piano tunes. These melodies were selected because they are less well known and for their simple structure. Only the melody lines were used.

The melodies were grouped into pairs. The melodies in each pair were roughly equated on a number of dimensions. Each melody pair had the same time signature, mode (major or minor), key, and tempo. Typically, paired melodies were originally written in different keys; therefore, a transposition of one of the melodies to the key of the other was necessary. Melodies were about 8 measures long, with the exception of melodies with a $2 / 4$ time signature, which were 16 measures long. All the melodies ended at the appropriate phrase ending. The tempo for each melody pair was determined by averaging the original tempos designated for the two melodies. 


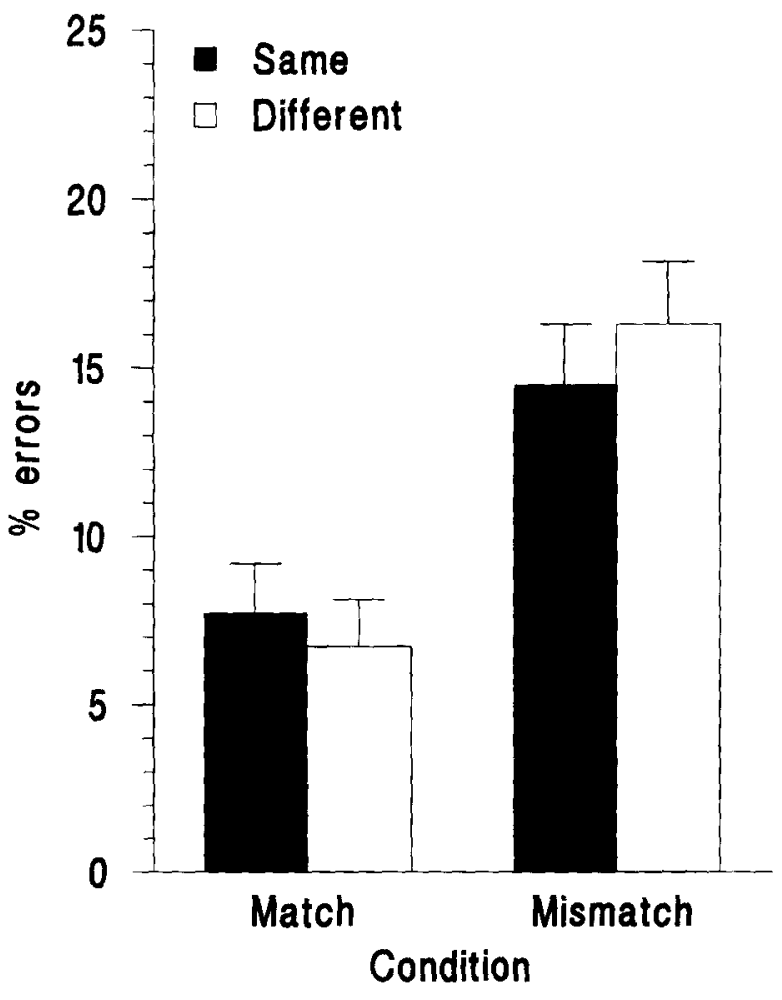

Figure 1. Error rate data (in percentages) for Experiment 1. Match/Mismatch refers to whether the target melody matched or mismatched the original melody in timbre. Same/Different refers to whether the distractor melody was in a timbre that was the same as or different from the original.

The pitch range between the melodies in a pair differed by $0-12$ halfsteps $(M=2.5, S D=2.6)$, deviated from each other in the number of accidentals by 0-7 $(M=1.4, S D=1.7)$, and varied in the number of rests by $0-5(M=1.0, S D=1.6)$. Although most melody pairs were the same in terms of the shortest and longest note values, 2 melody pairs differed in the length of the shortest note value, and 10 differed in the length of the longest note value (e.g., a quarter note vs. a dotted quarter). These deviations were largely confined to brief sections of the melodies.

The four timbres used were selected from the Waveblaster's bank of digitized sounds. These timbres were acoustic grand piano, vibraphone, English horn, and electric guitar (clean). The timbres were counterbalanced across the melodies so that each one appeared equally often. Because there were 32 melody pairs used, four of the timbre pair combinations were used five times, and two (acoustic grand piano-electric guitar (clean) and vibraphoneEnglish horn) were used six times. The melodies were encoded into the computer as MIDI files. The melodies were played at a constant dynamic and did not possess any timing irregularities.

Procedure. At the beginning of each session, the listeners filled out a musical history questionnaire that asked about any significant, prior experience in music performance or theory.

Melodies were presented via headphones at a comfortable listening level. The listeners were able to control the loudness. To initiate a trial, the listeners pressed the spacebar on the computer. At that time, the words "new melody" were displayed on the screen, and a recorded voice stated "melody" over the headphones. After this, the original melody was presented.
After each melody was presented, the listeners engaged in a 30sec distractor task. During this period, they were asked to solve a series of three-digit addition problems (e.g., $493+835=$ ?). This distractor task was included to encourage some forgetting of the original melody.

After the distractor task, the listeners saw and heard the words "Option 1" on the screen. Then, the first melody option was played. The same procedure was repeated for "Option 2." The task was to select which of the two melodies was the same as the original melody they had heard. After the completion of the second melody, the listeners chose their answer by pressing "l" or " 2 " on the keyboard. The computer recorded the responses. Across all trials, the order of the target and the distractor melodies was counterbalanced. For half the trials, the target preceded the distractor, and the reverse was true for the remainder. Furthermore, within each presentation order, the trials were counterbalanced with regard to the relationship of the original and the target melodies' timbres (match vs. mismatch) and with regard to the relationship of the target and the distractor melodies' timbres (same vs. different). The design is illustrated in Table 1.

Two practice trials were given to familiarize the listeners with the task and to provide them an opportunity to ask questions. The practice trials used the same procedure as the actual test. Listener responses on these trials were not recorded.

\section{Results and Discussion}

The error rate data for Experiment 1 are presented in Figure 1. As can be seen, more errors were made when the timbres of the target and the original melodies were mismatched $(15 \%)$ than when they were matched $(7 \%)$. This pattern in the different-timbre distractor condition replicates Radvansky et al. (1995) and Wolpert (1990). More generally, the data are consistent with the idea that timbre is being used as source information to select the memory trace. When the information matches, the trace can be more reliably retrieved than when it differs. The nature of the distractor had no influence on the memory decisions.

To confirm these observations, the error rate data were submitted to 2 (target type: match vs. mismatch) $\times 2$ (distractor type: same vs. different) repeated measures analyses of variance (ANOVAs). One ANOVA $\left(F_{1}\right)$ treated listeners as the random variable, and the other $\left(F_{2}\right)$ treated items as a random variable. There was a significant main effect of target type $\left[F_{1}(1,63)=35.42, M S_{\mathrm{e}}=121\right.$; $\left.F_{2}(1,31)=12.60, M S_{\mathrm{e}}=138\right]$. However, the main effect of distractor type and the interaction were not significant (all $F \mathrm{~s}<1$ ).

\section{EXPERIMENT 2 Pitch}

Experiment 1 established that people are able to effectively use a feature of an event, such as timbre, as a memory cue. When the feature is present, it is more likely that they will retrieve the memory trace. Our interpretation of this finding is that timbre is a memory cue of source information that is identified and used by people to access the memory trace. Simply put, timbre is an effective source cue. The purpose of Experiment 2 was to 


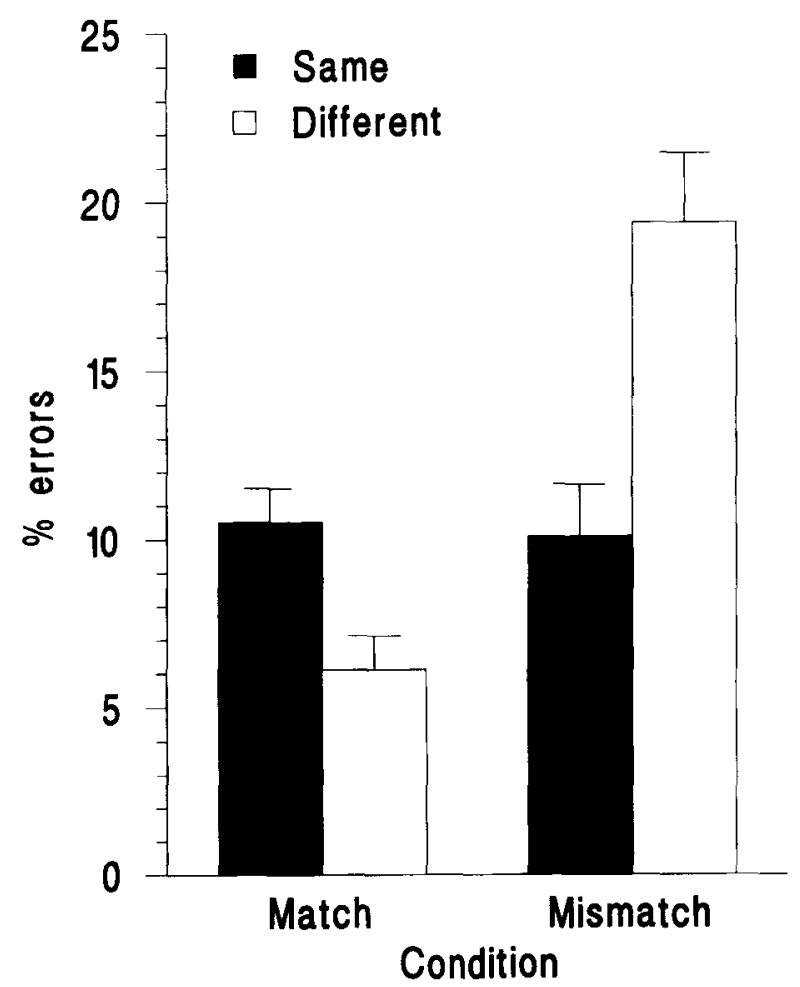

Figure 2. Error rate data (in percentages) for Experiment 2. Match/Mismatch refers to whether the target melody matched or mismatched the original melody in pitch. Same/Different refers to whether the distractor melody was in a pitch that was the same as or different from the original.

shore up this interpretation by showing that another feature of an event that is low-source information-namely, pitch - will be a poorer cue for the memory trace and will, therefore, show a different pattern of results.

\section{Method}

Listeners. Sixty-four people, with from 0 to 3 years $(M=1.3$, $S D=1.1$ ) of musical experience, were tested in Experiment 2 and given partial class credit in exchange for their participation.

Apparatus, Melodies, and Procedure. The apparatus, melodies, and procedure for Experiment 2 were identical to those in Experiment 1, with the exception that instead of manipulating timbre, pitch was manipulated. Piano was the only timbre that was used. On the pitch dimension, the alternative pitch differed from the original by an octave, a fifth, and a tritone.

\section{Results and Discussion}

The error rate data for Experiment 2 are presented in Figure 2. As can be seen, the pattern of error rates differed markedly from that for Experiment 1 . The fewest number of errors were made in the match-different condition $(6 \%)$. More errors were made in the match-same $(11 \%)$ and mismatch-same $(10 \%)$ conditions. Finally, the most errors were made in the mismatch-different condition $(19 \%)$. This is consistent with the idea that pitch is information that does not discriminate source well and is not used to recover the memory trace.
To confirm these observations, the error rate data were submitted to 2 (target type: match vs. mismatch) $\times 2$ (distractor type: same vs. different) repeated measures ANOVAs. There was a significant main effect of target type $\left[F_{1}(1,63)=20.24, M S_{\mathrm{e}}=130 ; F_{2}(1,31)=12.99\right.$, $\left.M S_{\mathrm{e}}=95\right]$. The main effect of distractor type was marginally significant $\left[F_{1}(1,63)=3.13, M S_{\mathrm{e}}=123, p=.08\right.$; $\left.F_{2}(1,31)=3.34, M S_{\mathrm{e}}=77, p=.08\right]$. Finally, the interaction was also significant $\left[F_{1}(1,63)=24.40, M S_{\mathrm{e}}=123\right.$; $\left.F_{2}(1,31)=20.13, M S_{\mathrm{e}}=65\right]$.

In order to verify the differences between Experiments 1 and 2, the error rate data for the two experiments were submitted to 2 (experiment) $\times 2$ (target type: match vs. mismatch) $\times 2$ (distractor type: same vs. different) mixed ANOVAs. Importantly, the three-way interaction was significant $\left[F_{1}(1,126)=7.39, M S_{\mathrm{e}}=124 ; F_{2}(1,62)=\right.$ $\left.7.46, M S_{\mathrm{e}}=93\right]$. This is consistent with the idea that highsource and low-source information have different consequences when they are available for use as memory cues.

Of less central interest, there was a significant main effect of target type $\left[F_{1}(1,126)=54.64, M S_{\mathrm{e}}=126\right.$; $\left.F_{2}(1,62)=25.32, M S_{\mathrm{e}}=116\right]$ and a significant target type $\times$ distractor type interaction $\left[F_{1}(1,126)=17.10\right.$, $\left.M S_{\mathrm{e}}=124 ; F_{2}(1,62)=6.54, M S_{\mathrm{e}}=93\right]$. All other $F \mathrm{~s} \leq$ 1.01 , except for the main effect of distractor type in the listeners analysis $\left[F_{1}(1,126)=2.02, M S_{\mathrm{e}}=244, p=.16\right]$ and the experiment $\times$ distractor type interaction in the items analysis $\left[F_{2}(1,62)=2.10, M S_{\mathrm{e}}=91, p=.15\right]$.

\section{EXPERIMENT 3}

The purpose of Experiment 3 was to gain some insight into the degree to which source cuing is influenced by retrieval context. Specifically, in Experiment 1, timbre could be perceived as being a useful diagnostic by the retrieval system because there were several cases in which the target and the distractor differed in their timbre. The same was true in the Radvansky et al. (1995) and Wolpert (1990) studies, which showed a similar pattern between match and mismatch conditions.

There are two general possibilities to be considered here. First, if source cuing operates independently of context, it can be expected that even when the two probe options share the same timbre, there will be a difference in memory performance. People will be better when the options match the timbre of the original than when they mismatch, much like what was observed in the sametimbre conditions of Experiment 1 . The second possibility is that source cuing only has an appreciable effect when there are a number of trials present on which the options differ in terms of timbre. If the target and the distractor melodies were always in the same timbre, people would be less likely to use timbre information in making a selection, because it would no longer be a salient dimension along which the items could be discriminated and so would function less efficiently as a retrieval cue. The prediction is that, in this situation, performance will be the same for the match and the mismatch conditions. 


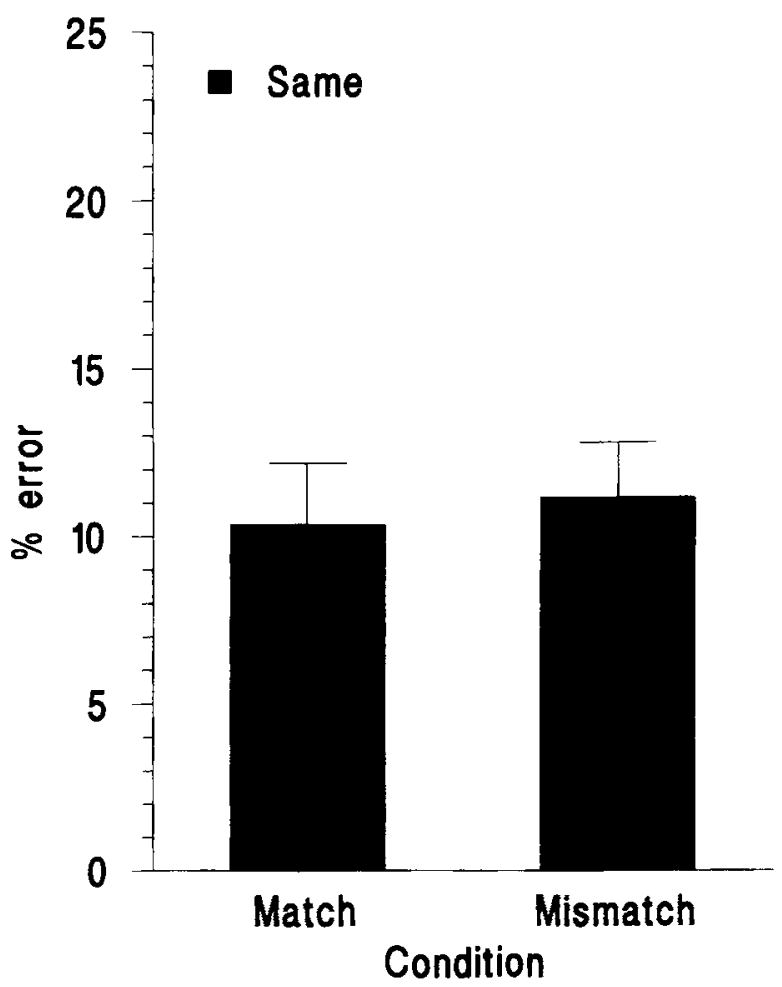

Figure 3. Error rate data (in percentages) for Experiment 3. Match/Mismatch refers to whether the target melody matched or mismatched the original melody in timbre.

\section{Method}

Listeners. Thirty-two people, with from 0 to 3 years $(M=1.2$, $S D=1.1)$ of musical experience, were tested in Experiment 3 and given partial class credit in exchange for their participation.

Apparatus, Melodies, and Procedure. The apparatus, melodies, and procedure were the same as those used in Experiment 1 , with the exception that the timbre of the target and the distractor melodies was always the same (second and fourth columns in Table 1).

\section{Results and Discussion}

The error rate data for Experiment 3 are presented in Figure 3. Three listeners in Experiment 3 made no errors. As can be seen, the pattern of error rates differed from that for Experiment 1. Specifically, a similar number of errors were made in the match $(10 \%)$ and mismatch $(11 \%)$ conditions. This is consistent with the idea that source information, such as timbre, can be used to aid recognition decisions but is likely to be given more weight when it can be used to discriminate between alternatives, such as when they differ along this dimension. In order for the source cuing effects to be observed, this information needs to be manipulated across trials so that there are a fair number of times when the target and the distractor differ on this dimension.

To confirm these observations, the error rate data were submitted to (target type: match vs. mismatch) repeated measures ANOVAs. There were no significant effects (both $F \mathrm{~s}<1$ ). This is in contrast to the pattern of data observed in the match-same and mismatch-same conditions of Experiment 1. To confirm this idea, the error rate data were submitted to 2 (experiment) $\times 2$ (target type: match vs. mismatch) mixed ANOVAs. Only the matchsame and mismatch-same data were used from Experiment 1 . Of most interest, the experiment $\times$ target type interaction was marginally significant $[F(1,94)=3.71$, $\left.M S_{\mathrm{e}}=103, p=.057\right]$.

\section{GENERAL DISCUSSION}

The purpose of the present study was to assess whether people treat event features differently with regards to the feature's ability to indicate source information, as well as to assess a person's ability to use this information in retrieval. High-source features were better retrieval cues than low-source features. This increased effectiveness is referred to as source cuing.

It was found that a feature, such as a melody's timbre, can serve effectively as a source cue. When people had to indicate which of two melodies was heard earlier, they were more accurate when the timbre of the target melody matched the original than when it mismatched (Experiment 1 ). In contrast, it was also found that other features, such as pitch, may be less effective source cues (Experiment 2). This suggests that high-source features can be used to help access the memory trace for that event, allowing for a more accurate selection. However, such source cues are likely to be given more weight in retrieval when they vary and can be used to help discriminate among the options (Experiment 3). This research is also consistent with the idea that item and source information are processed differently (e.g., Senkfor \& Van Patten, 1998). In the present experiments, the melody served as the item information, since it was the defining information that needed to be retrieved. The source information was provided, in the context of the present experiments, by the timbre through which the melody was played. It can be seen that these two types of information were processed differently, in that the use of item information alone to discriminate alternatives resulted in a different pattern of responding (Experiment 3 ) than was found when the source information was available in addition (Experiment 1).

From the data presented here, it is clear that some features are better for discriminating memory traces than others. We have suggested that a dimension that regulates this effectiveness is the degree to which the feature can be used to discriminate between various sources. What leads to this? One possible explanation borrows from Chalfonte and Johnson (1996) the idea of feature binding. According to this idea, different types of information (e.g., features) can be associated with the identity of a stimulus. These information types can differ in terms of how strongly they are bound with the memory of an event. By 
definition, features that are more strongly bound are more likely to be linked with the item information in the memory trace. In contrast, weakly bound features are less likely to be linked with the item information in longterm memory. On this view, high-source information would be more likely to be bound with a memory trace than low-source information (all else being equal) would be. Thus, high-source features are more effective retrieval cues in accessing a memory trace that actually contains the melody information. Other low-source features may carry qualitative information about the stimulus that is of less importance along this dimension. As a result, they are less effective when used.

The present experiments may also be consistent with the idea that, over time, there is a forgetting of stimulus attributes (e.g., Riccio et al., 1994). The rate at which these attributes are forgotten varies, depending on the nature of the attribute. For example, attributes related to source are forgotten at a more rapid rate than attributes related to frequency. From this view, the results of Experiments 1 and 2 reflect the more rapid forgetting of pitch, relative to timbre, causing pitch to be a poorer memory cue. It should be noted that it is possible that the origin of this differential forgetting may still be related to the degree to which the attribute conveys information about the source. That is, high-source features are better stored and retained, relative to low-source features.

In general, the present study further illustrates that source information is important for memory. It is already well established how people retrieve and report the source of information in memory. The present study shows that source information can also be used to cue the retrieval of a desired memory trace. Some ideas about how this may be related to feature binding or to attribute forgetting have been discussed. However, further effort is needed to address these issues.

\section{REFERENCES}

Agnay, D. (ED.) (1956). Easy classics to moderns. New York. Consolidated Music Publishers.

Chalfonte, B. L., \& Johnson, M. K. (1996). Feature memory and binding in young and older adults. Memory \& Cognition, 24, 403416.

HaRTMAnN, W. M., \& Johnson, D. (1991). Stream segregation and peripheral channeling. Music Perception, 9, 155-183.

JoHnson, M. K., HaShTroudi, S., \& Lindsay, D. S. (1993). Source monitoring. Psychological Bulletin, 114, 3-28.

Mullennix, J. W., Pisoni, D. B., \& Martin, C. S. (1989). Some effects of talker variability on spoken word recognition. Journal of the Acoustical Society of America, 85, 365-378.

Palmeri, T. J., Goldinger, S. D., \& Pisoni, D. B. (1993). Episodic encoding of voice attributes and recognition memory for spoken words. Journal of Experimental Psychology: Learning, Memory, \& Cognition, 19, 309-328.

Peretz, I., Gaudreau, D., \& Bonnel, A.-M. (1998). Exposure effects on music preference and recognition. Memory \& Cognition, 26, 884902.

Radvansky, G. A., Fleming, K. J., \& Simmons, J. A. (1995). Timbre reliance in nonmusicians' and musicians' memory for melodies. Music Perception, 13, 127-140.

Riccio, D. C., Rabinowitz, V. C., \& Axelrod, S. (1994). Memory: When less is more. American Psychologist, 49, 917-926.

SEnkfor, A. J., \& VAN PATten, C. (1998). Who said what? An eventrelated potential investigation of source and item memory. Journal of Experimental Psychology: Learning, Memory, \& Cognition, 24, 1005-1025.

Tulving, E., \& Thompson, D. M. (1973). Encoding specificity and retrieval processes in episodic memory. Psychological Review, 80, 352373.

WOLPERT, R. S. (1990). Recognition of melody, harmonic accompaniment, and instrumentation: Musicians and nonmusicians. Music Perception, 8, 95-106.

\section{NOTE}

1. Of course, if the pitch were to change by a large amount, such as more than an octave, it would become more likely that this could be used to discriminate a source. However, it should also be kept in mind that larger shifts in pitch also have more noticeable influences on the perceived timbre. Thus, a relatively restricted pitch range was used here.

(Manuscript received June 16, 1998; revision accepted for publication July 29,1999 .) 